\title{
Dimension Estimate for the Global Attractor of an Evolution Equation
}

\author{
Renato Colucci and Gerardo R. Chacón \\ Departamento de Matemática, Pontificia Universidad Javeriana, Carrera 7 No. 43-82, Bogotá, Colombia
}

Correspondence should be addressed to Renato Colucci, renatocolucci@hotmail.com

Received 29 September 2011; Accepted 13 December 2011

Academic Editor: Juan J. Nieto

Copyright (c) 2012 R. Colucci and G. R. Chacon. This is an open access article distributed under the Creative Commons Attribution License, which permits unrestricted use, distribution, and reproduction in any medium, provided the original work is properly cited.

We estimate the dimension of the global attractor of an evolution equation by the study of the evolution of the $n$-dimensional volumes under the flow. We compare these results with the estimate of the dimension of the inertial manifold.

\section{Introduction}

One of the most interesting problems in the analysis of partial differential equations is the study of the asymptotic behavior of solutions. Guided by the finite-dimensional case, in the last decades, the concept of global attractor was introduced in order to study the long-term dynamics of dissipative equations (see, e.g., $[1,2]$ and the references therein). This theory has been generalized to the case on non-autonomous systems by introducing the definition of uniform attractor; see, for example, the interesting article [3] in which a reaction diffusion equation is studied with nonlinear boundary conditions in competitions with the dissipative terms. However, the existence of uniform attractors fails in many cases and that is the reason why the new definition of Pullback Attractor is introduced (see [4]). This new concept works for both the non-autonomous (see [5]) and the random systems, for parabolic (see [6]), and hyperbolic stochastic equations (see [7]) in bounded and in unbounded domains. A further generalization of the concept of attractor to the setting of multivalued processes can be seen in [8], and an application to the case of 3D Navier Stokes systems can be seen in [9].

Once the existence of a global attractor has been established, in order to analyze its structure, it is a common question to investigate the (fractal) dimension of it. The classical method to do that consists in the study of the contraction of an $n$-dimensional volume element under the action of the flow (see [1, 2]). This is a very sharp method that works also in the case of non-autonomous systems; see, for instance, [5] in which also is 
pointed out the influence of the geometry of the domain in determining the dimension of attractors.

This method also works in numerical context; in fact there are also numerical schemes (see, e.g., [10]) that preserve important properties of the solution and that preserve the dimension of the attractor (see, for instance, [11]).

The disadvantage of the study of volume contraction method is that it requires higher regularity of the data and the solutions this can be avoided by using the theory of exponential attractors (see [12]) or the so-called method of l-trajectories (see, e.g., [13]). Both methods require less regularity and prove finite dimension of the attractor.

In this paper, we will study the dimension of the global attractor of an autonomous evolution equation by using different methods. The autonomous evolution equation considered here is a simple model of a dynamical system which presents different time scales in which the dynamics presents different features. We consider the following fourth-order evolution equation:

$$
\begin{gathered}
u_{t}=-\varepsilon^{2} u_{x x x x}+\frac{1}{2} \Phi^{\prime \prime}\left(u_{x}\right) u_{x x}, \quad(x, t) \in I \times \mathbb{R}_{+}, \\
u(0, x)=u_{0}(x), \quad \text { in } I, \\
u=u_{x x}=0, \quad \text { in } \partial I \times \mathbb{R}_{+},
\end{gathered}
$$

where the function $\Phi(p)=\left(p^{2}-1\right)^{2}$ is the so-called double well potential and $I \subset \mathbb{R}$ is an open interval such that its length $|I| \leq 1$. In particular, (1.1) is the $L^{2}$-gradient flow associated to the functional:

$$
F_{\varepsilon}(u)=\frac{1}{2} \varepsilon^{2} \int_{I} u_{x x}^{2} d x+\frac{1}{2} \int_{I} \Phi\left(u_{x}\right) d x
$$

In [14] it is studied the global dynamics of an evolution equation like (1.1) with a more general nonconvex function $\Phi$. They use numerical experiments to discuss the dynamical behavior of the solutions for small values of $\varepsilon$. In particular it was pointed out the existence of three different time scales with peculiar dynamic behavior.

In a first time scale of order $t>T_{\varepsilon}=O\left(\varepsilon^{2}\right)$, there is a drastic reduction of the energy $F_{\varepsilon}\left(u_{0}\right)$ of the initial data $u_{0}$ and a formation of microstructure in the region where $\Phi(\cdot)$ is not convex.

In the second time scale of order $t>T=O(1)$, we have that the equation exhibits a heat equation-like behavior in the convex regions while slow motion in the nonconvex ones.

In the third time scale of order $t>1 / T_{\varepsilon}=O\left(\varepsilon^{-2}\right)$, the infinite dynamical system is reduced to a finite one. The solution is approximately the union of consecutive segments. The dynamic is slow and the number of segments decreases letting decrease the number of freedom degrees of the problem.

We use the following notation throughout the paper. We develop the analysis in the following spaces:

$$
H=L^{2}(I), \quad V=H^{2}(I) .
$$


In [15] the asymptotic behavior of (1.1) was studied and it was proven the existence of a global attractor for the semigroup associated to (1.1), that is, the semigroup of operators defined as

$$
S(t): u_{0} \in H \longrightarrow u(t) \in H,
$$

The main tool in proving the existence of a global attractor is to show the existence of an absorbing set in $H^{2}(I)$ (see Theorem 1.1 in [1]).

Theorem 1.1 (see [15]). There exist positive constants $r_{i}, \tau_{i}$ with $i=0,1,2$ such that

(i) the semigroup $S(t)$ posses an absorbing set in $H$ :

$$
\left\|S(t) u_{0}\right\| \leq r_{0}, \quad \text { if } t \geq \tau_{0},
$$

where

$$
r_{0}=\left(1+\frac{|I|}{\varepsilon^{2}}\right)^{1 / 2}, \quad \tau_{0}=\frac{1}{\varepsilon^{2}}\left\|u_{0}\right\|
$$

(ii) the semigroup $S(t)$ posses an absorbing set in $H_{0}^{1}(I)$ :

$$
\left\|S(t) u_{0}\right\|_{H_{0}^{1}(I)} \leq r_{1}, \quad \text { if } t \geq \tau_{1}
$$

where

$$
r_{1}=\left\{r_{0}^{2}\left(1+\frac{1}{2 \varepsilon^{4}}\right)+\frac{|I|}{\varepsilon^{2}}\right\}^{1 / 2} e, \quad \tau_{1}=\tau_{0}+\varepsilon^{2}
$$

(iii) the semigroup $S(t)$ posses an absorbing set in $H^{2}(I)$ :

$$
\left\|S(t) u_{0}\right\|_{H^{2}(I)} \leq\left(r_{0}^{2}+r_{1}^{2}+r_{2}^{2}\right)^{1 / 2}, \quad \text { if } t \geq \tau_{2}
$$

where

$$
r_{2}=\frac{e^{2}}{\varepsilon^{2}}\left\{180 r_{1}^{6}+\frac{r_{0}^{2}}{2}+|I| \varepsilon^{2}\right\}^{1 / 2}, \quad \tau_{2}=\tau_{1}+\varepsilon^{2}
$$

Theorem 1.1 let us conclude that the set

$$
\mathbb{B}_{\varepsilon}=\left\{u \in H:\left\|u_{x}\right\| \leq r_{1},\left\|u_{x x}\right\| \leq r_{2}\right\}
$$


is absorbing for all the bounded sets of $H$. In particular the global attractor is given as the omega limit set of the absorbing set, $A_{\varepsilon}=\omega(\mathbb{B})$. The authors also obtained in [15] a bound for the dimension of $\mathcal{A}_{\varepsilon}$ by proving the existence of an exponential attractor $\supset_{\varepsilon}$ whose dimension is of order $O\left(\varepsilon^{-10}\right)$. Moreover, in $\boldsymbol{B}_{\varepsilon}$ we have the following estimates for $t>\tau_{2}$ :

$$
\begin{gathered}
\|u\|_{\infty} \leq\|u\|^{1 / 2}\left\|u_{x}\right\|^{1 / 2} \leq\left(r_{0} r_{1}\right)^{1 / 2}, \\
\left\|u_{x}\right\|_{\infty} \leq\left\|u_{x}\right\|^{1 / 2}\left\|u_{x x}\right\|^{1 / 2} \leq\left(r_{1} r_{2}\right)^{1 / 2} .
\end{gathered}
$$

In accordance with the numerical experiments, the time in which the bounded solutions enter the absorbing set is of order $O\left(\varepsilon^{-2}\right)$ (see $[14,15]$ ).

We are interested in finding estimates for the dimension of $\mathcal{A}_{\varepsilon}$. In Section 2 we find an estimate of the fractal and Hausdorff dimension of $A_{\varepsilon}$ by studying the evolution of $n$ dimension volume elements, this following a classical theory by Temam (see [1]). In Section 3 we prove the existence of an inertial manifold $\boldsymbol{M}_{\varepsilon}$ (see [16]) for the system (1.1), that is, a smooth manifold that attracts all the orbit at an exponential rate and that contains the global attractor. By estimating the dimension of $\mathcal{M}_{\varepsilon}$, we obtain, in another way, a different estimate of $\operatorname{dim}\left(\mathcal{A}_{\varepsilon}\right)$. In Section 4 we study the regularity of the attractor while in the last section we prove the existence of an absorbing set in $L^{\infty}(I)$. This is fundamental information in order to study the approximation of the attractor (see [17]).

We define the linear operator $A=\partial^{4} / \partial x^{4}$. The set $D(A)=\left\{u \in H^{4}(I): u=u_{x x}=\right.$ 0 in $\partial I\}$ is its domain. Also, then $(A u, u)=\left\|u_{x x}\right\|^{2}$ where $\|\cdot\|:=\|\cdot\|_{L^{2}(I)}$ and the operator is self-adjoint in $D(A)$. From classical results on solutions of evolution equations (see [18] or see Theorems 3.1 and 3.3 on [1]), we have the following:

Theorem 1.2. Problem (1.1) admits a unique solution $u$ such that

$$
\begin{array}{cc}
\text { if } u_{0} \in H \Longrightarrow u \in C^{0}([0, T], H) \cap L^{2}(0, T ; V), & \forall T>0 ; \\
\text { if } u_{0} \in V \Longrightarrow u \in C([0, T], V) \cap L^{2}(0, T ; D(A)), & \forall T>0 .
\end{array}
$$

\section{Dimension of the Attractor}

Following $[1,2]$ we give an estimate of the dimension of the attractor $\mathcal{A}_{\varepsilon}$ by estimating how the $n$-dimensional volume elements are distorted by the flow. We will show the following theorem

Theorem 2.1. The global attractor $\mathcal{A}_{\varepsilon}$ of the semigroup has fractal dimension $d_{F}\left(\mathcal{A}_{\varepsilon}\right)<2 n$ where $n: O\left(\varepsilon^{-1}\right)$.

Proof. In general if we consider an evolution equation of the type

$$
\begin{aligned}
& u_{t}=F(u), \\
& u(0)=u_{0},
\end{aligned}
$$

then the idea is to investigate the evolution of $n$-dimensional infinitesimal volumes and find the smallest $n$ such that all the $n$-infinitesimal volumes contract exponentially. Then one may 
guess that the attractor does not contain these elements and so that the dimension of $\mathcal{A}_{\varepsilon}$ is less than or equal to $n$. We study the evolution of a set of infinitesimal displacement $\delta x^{(i)}$ about a trajectory $v(t)$. In order to do that, we linearize the equation around the solution $v(t)=S(t) u_{0}:$

$$
\begin{gathered}
U_{t}=F^{\prime}\left(S(t) u_{0}\right) U:=L\left(t ; u_{0}\right) U, \\
U(0)=\xi,
\end{gathered}
$$

where $F^{\prime}$ is the Fráchet derivative of $F$. Each displacement evolves

$$
\frac{d}{d t} \delta x^{(i)}=L\left(t ; u_{0}\right) \delta x^{(i)}
$$

The volume is given by $V_{n}(t)=\left|\delta x^{(1)} \wedge \cdots \wedge \delta x^{(n)}\right|$ where

$$
\left|\delta x^{(1)} \wedge \cdots \wedge \delta x^{(n)}\right|^{2}=\operatorname{det}\left(\left(\delta x^{(i)}, \delta x^{(j)}\right)\right)_{i, j}:=\operatorname{det} M(t),
$$

then (see, e.g., [2] for details)

$$
\frac{d}{d t} V_{n}(t)=\frac{1}{2} T R\left[M^{-1} \frac{d}{d t} M\right]
$$

Then if $P_{n}(t)$ is the projector on the space spanned by the displacements $\delta x^{(i)}$ with base $\varphi_{1}(t), \ldots, \varphi_{n}(t)$, we have

$$
V_{n}(t)=V_{n}(0) \exp \left(\int_{0}^{t} T R\left(L\left(s ; u_{0}\right) P_{n}(s)\right) d s\right)
$$

and consequently the asymptotic growth rate is given by

$$
\lim _{t \rightarrow \infty} \frac{1}{t} \int_{0}^{t} T R\left(L\left(s ; u_{0}\right) P_{n}(s)\right) d s .
$$

As we need the maximal growth rate, we compute the sup over the $u_{0} \in \mathcal{A}_{\varepsilon}$ and all the n-dimensional projectors:

$$
\sup _{u_{0} \in \mathscr{A}_{\varepsilon}} \sup _{P_{n}} \limsup _{t \rightarrow+\infty} \frac{1}{t} \int_{0}^{t} T R\left(L\left(s ; u_{0}\right) P_{n}(s)\right) d s:=T R_{n}\left(\mathscr{A}_{\varepsilon}\right) .
$$

In order to have exponential decay, we look for the smallest $n$ such that the number $T R_{n}\left(\mathcal{A}_{\varepsilon}\right)$ is negative. Thus, using theorem 13.16 page 341 in [2] let us conclude that

$$
d_{F}\left(\mathcal{A}_{\varepsilon}\right) \leq 2 n
$$


In order to estimate $T R_{n}\left(\mathcal{A}_{\varepsilon}\right)$, we look for an uniform bound of the following expression:

$$
\sup _{u_{0} \in \mathcal{A}} \sup _{P_{n}} T R\left(L\left(s ; u_{0}\right) P_{n}(s)\right) d s \leq C, \quad \forall s,
$$

from which we conclude that $T R_{n}(\mathcal{A}) \leq C$. In our case the linearized equation is

$$
\begin{gathered}
U_{t}=-\varepsilon^{2} U_{x x x x}-2 U_{x x}+6 v_{x}^{2} U_{x x}+12 v_{x} v_{x x} U_{x} . \\
U(0)=\xi .
\end{gathered}
$$

In details let $\varphi_{1}(t), \ldots, \varphi_{n}(t)$ be an orthonormal base for $P_{n} L^{2}(I)$ then

$$
\operatorname{TR}\left(F^{\prime}(v) P_{n}\right)=\sum_{i=1}^{n}\left(F^{\prime}(v) \varphi_{i}, \varphi_{i}\right)_{L^{2}(I)^{\prime}}
$$

with $v=S(t) u_{0}$,

$$
F^{\prime}(v)=-\varepsilon^{2} D_{x x x x}-2 D_{x x}+6 v_{x}^{2} D_{x x}+12 v_{x} v_{x x} D_{x} .
$$

We estimate the terms separately. The first term

$$
-\varepsilon^{2} \sum_{i=1}^{n}\left(D_{x x x x} \varphi_{i}, \varphi_{i}\right)=-\varepsilon^{2} \sum_{i=1}^{n}\left\|\varphi_{i x x}\right\|^{2}
$$

The second term

$$
-2\left(D_{x x} \varphi_{i}, \varphi_{i}\right)=2\left\|\varphi_{i x}\right\|_{L^{2}(I)^{\prime}}^{2}
$$

from which by interpolation we get

$$
\begin{aligned}
-2 \sum_{i=1}^{n}\left(D_{x x} \varphi_{i}, \varphi_{i}\right) & =2 \sum_{i=1}^{n}\left\|\varphi_{i x}\right\|_{L^{2}(I)}^{2} \leq 2 \sum_{i=1}^{n}\left\|\varphi_{i}\right\|\left\|\varphi_{i x x}\right\| \\
& \leq 2\left(\sum_{i=1}^{n}\left\|\varphi_{i}\right\|^{2}\right)^{1 / 2}\left(\sum_{i=1}^{n}\left\|\varphi_{i x x}\right\|^{2}\right)^{1 / 2}=2 n^{1 / 2}\left(\sum_{i=1}^{n}\left\|\varphi_{i x x}\right\|^{2}\right)^{1 / 2} \\
& \leq \frac{\varepsilon^{2}}{2} \sum_{i=1}^{n}\left\|\varphi_{i x x}\right\|^{2}+\frac{2 n}{\varepsilon^{2}}
\end{aligned}
$$

The last term

$$
\sum_{i=1}^{n}\left\{6 \int_{I} v_{x}^{2} \varphi_{i x x} \varphi_{i} d x+12 \int_{I} v_{x} v_{x x} \varphi_{i x} \varphi_{i} d x\right\}=-\sum_{i=1}^{n}\left\{6 \int_{I} v_{x}^{2} \varphi_{i x}^{2} d x\right\}
$$


is negative and consequently it can be neglected in the computation. Then we have the following estimate:

$$
\begin{aligned}
\operatorname{TR}\left(F^{\prime}(v) P_{n}\right) & \leq-\varepsilon^{2} \sum_{i=1}^{n}\left\|\varphi_{i x x}\right\|^{2}+\frac{\varepsilon^{2}}{2} \sum_{i=1}^{n}\left\|\varphi_{i x x}\right\|^{2}+\frac{2 n}{\varepsilon^{2}} \\
& =-\frac{\varepsilon^{2}}{2} \sum_{i=1}^{n}\left\|\varphi_{i x x}\right\|^{2}+\frac{2 n}{\varepsilon^{2}} \leq-\frac{\varepsilon^{2}}{2} \lambda_{1} n^{5}+\frac{2 n}{\varepsilon^{2}},
\end{aligned}
$$

where we have used that

$$
-\sum_{i=1}^{n}\left(D_{x x x x} \varphi_{i}, \varphi_{i}\right) \leq-\lambda_{1} n^{5}
$$

and where $\lambda_{j}=\left((2 \pi)^{4} /|I|^{4}\right) j^{4}=\lambda_{1} j^{4}$ are the eigenvalues of the operator $D_{x x x x}$. Since from (2.18) we have that $T R\left(F^{\prime}(v) P_{n}\right)$ is uniformly bounded in time, then we conclude that

$$
T R_{n}\left(\mathcal{A}_{\varepsilon}\right) \leq-k_{1} n^{5}+k_{2}
$$

where we have used a Young inequality and the constants $k_{1}, k_{2}$ are given by

$$
\begin{aligned}
& k_{1}=\frac{\varepsilon^{2} \lambda_{1}}{4}, \\
& k_{2}=\frac{2^{15 / 4}}{\varepsilon^{3} \lambda_{1}^{1 / 4} 5^{5 / 4}} .
\end{aligned}
$$

This (see [1]) also gives an estimate for the Lyapunov exponents $\mu_{i}$ :

$$
\mu_{1}+\cdots+\mu_{n} \leq T R_{n}\left(\mathscr{A}_{\varepsilon}\right) \leq-k_{1} n^{5}+k_{2}, \quad \forall n \in \mathcal{N}
$$

Moreover (see [1], Theorem 2.2 page. 396), we obtain an estimate on the dimension of $A_{\varepsilon}$.

Theorem 2.2. Let $n \in \mathcal{N}$ such that

$$
n-1<\left(\frac{2 k_{2}}{k_{1}}\right)^{1 / 5} \leq n
$$

then the $n$-dimensional volume element in the phase space is exponentially decaying as $t \rightarrow \infty$. Moreover, the global attractor $\mathscr{A}_{\varepsilon}$ has finite dimension: $d_{H}\left(\mathscr{A}_{\varepsilon}\right) \leq n, d_{F}\left(\mathscr{A}_{\varepsilon}\right) \leq 2 n$.

Then we have that

$$
n \sim\left[\frac{2^{7 / 20}|I|}{\pi 5^{1 / 4}}\right] \varepsilon^{-1} .
$$


From the above expression we have that $n=O\left(\varepsilon^{-1}\right)$. This provides a much better estimate than the one obtained in [15] by the fractal dimension of the exponential attractor $n_{*}=O\left(\varepsilon^{-10}\right)$.

\section{Estimate via the Existence of the Inertial Manifold}

In this section we will show the existence of the inertial manifold $\mathcal{M}_{\varepsilon}$ for system (1.1). Then the dimension of $\mathcal{M}_{\varepsilon}$ will provide a further estimate of the dimension of the global attractor since $A_{\varepsilon} \subset \mathcal{M}_{\varepsilon}$. In order to carry on our analysis, we follow the strategy explained in [2].

Theorem 3.1. The system (1.1) possesses an inertial manifold $\boldsymbol{M}_{\varepsilon}$ having dimension of order $O\left(\varepsilon^{-19}\right)$.

One first restricts (1.1) to the absorbing set $\boldsymbol{B}_{\varepsilon}$. Then in order to prove the existence of $\mathcal{M}_{\varepsilon}$ one has to check the fulfillment of two conditions. The first condition consists of proving that the nonlinear term $R(u):=(-1 / 2) \Phi^{\prime \prime}\left(u_{x}\right) u_{x x}$ of $(1.1)$ is Lipschitz from $H^{2}(I)$ to $L^{2}(I)$ :

$$
\left\|R\left(u_{1}\right)-R\left(u_{2}\right)\right\| \leq C_{L}\left\|u_{1}-u_{2}\right\|_{H^{2}(I)},
$$

$\forall u, v \in \mathbb{B}_{\varepsilon} \cap H^{2}(I)$. In particular the constant $C_{L}=C_{L}\left(\mathbb{B}_{\varepsilon}\right)$ depends on the radius of $\mathbb{B}_{\varepsilon}$.

The second condition is the so-called strong squeezing property.

Definition 3.2. Let $u_{1}, u_{2}$ two solutions of (1.1), the strong squeezing property holds if for some $N$ and $K$

$$
\left\|Q_{N}\left(u_{1}(0)-u_{2}(0)\right)\right\|_{H^{2}(I)} \leq K\left\|P_{N}\left(u_{1}(0)-u_{2}(0)\right)\right\|_{H^{2}(I)}
$$

implies that

$$
\left\|Q_{N}\left(u_{1}(t)-u_{2}(t)\right)\right\|_{H^{2}(I)} \leq K\left\|P_{N}\left(u_{1}(t)-u_{2}(t)\right)\right\|_{H^{2}(I)}, \quad \forall t \geq 0,
$$

and furthermore that if

$$
\left\|Q_{N}\left(u_{1}\left(t_{0}\right)-u_{2}\left(t_{0}\right)\right)\right\|_{H^{2}(I)} \geq K\left\|P_{N}\left(u_{1}\left(t_{0}\right)-u_{2}(t)_{0}\right)\right\|_{H^{2}(I)}
$$

then

$$
\left\|Q_{N}\left(u_{1}(t)-u_{2}(t)\right)\right\|_{H^{2}(I)} \leq\left\|Q_{N}\left(u_{1}(0)-u_{2}(0)\right)\right\|_{H^{2}(I)} e^{-\tilde{k} t},
$$

for $0 \leq t \leq t_{0}$ and some $\tilde{k}>0$ and where $Q_{N}=I-P_{N}$.

Again following [2] we have that a sufficient condition to prove that the strong squeezing property holds is the so-called spectral gap condition:

$$
\tilde{\lambda}_{N+1}-\tilde{\lambda}_{N}>2 C_{L}\left(\tilde{\lambda}_{N+1}^{1 / 2}+\tilde{\lambda}_{N}^{1 / 2}\right)
$$


for $N$ sufficiently large and where $\tilde{\lambda}_{k}$ are the eigenvalues of the operator $\tilde{A}=\varepsilon^{2} D_{x x x x}$. The spectral gap condition will be the second property we will show holds. We divide the proof into two steps.

\subsection{Lipschitz Property for $R$}

Theorem 3.3. Given solutions $u_{1}$ and $u_{2}$, then there exists a constant $C_{L}>0$ such that

$$
\left\|R\left(u_{1}\right)-R\left(u_{2}\right)\right\|_{L^{2}(I)} \leq C_{L}\left\|u_{1}-u_{1}\right\|_{H^{2}(I)},
$$

where $R$ denotes the nonlinear part of (1.1), that is,

$$
R(u):=-\frac{1}{2} \phi^{\prime \prime}\left(u_{x}\right) u_{x x}
$$

Proof. First, we suppose that $u$ is a solution of (1.1), then, for any unitary vector $v$ in $L^{2}(I)$, we have that

$$
\begin{aligned}
\langle R(u), v\rangle & =\int_{I}-\frac{1}{2} \phi^{\prime \prime}\left(u_{x}(s)\right) u_{x x}(s) v(s) d s \\
& =2 \int_{I}\left(1-3 u_{x}^{2}(s)\right) u_{x x}(s) v(s) d s \\
& =2 \int_{I} u_{x x}(s) v(s) d s-6 \int_{I} u_{x}^{2}(s) u_{x x}(s) v(s) d s \\
& \leq 2\left\|u_{x x}\right\|_{L^{2}(I)}\|v\|_{L^{2}(I)}+6\left\|u_{x}\right\|_{\infty}^{2}\left\|u_{x x}\right\|_{L^{2}(I)}\|v\|_{L^{2}(I)} \\
& =\left(2+6\left\|u_{x}\right\|_{\infty}^{2}\right)\|v\|_{L^{2}(I)}\left\|u_{x x}\right\|_{L^{2}(I)} \\
& \leq\left(2+6\left\|u_{x}\right\|_{\infty}^{2}\right)\|v\|_{L^{2}(I)}\|u\|_{H^{2}(I)}
\end{aligned}
$$

and consequently the nonlinear operator restricted on $\boldsymbol{B}_{\varepsilon}$ is bounded from $H^{2}(I) \rightarrow L^{2}(I)$ :

$$
\|R(u)\|_{L^{2}(I)} \leq\left(2+6 r_{1} r_{2}\right)\|u\|_{H^{2}(I)} .
$$


Now, if $u_{1}$ and $u_{2}$ are solutions of (1.1), let $u:=u_{1}-u_{2}$, then we proceed in a similar manner as before to get that if $v \in L^{2}(I)$, then

$$
\begin{aligned}
\left\langle R\left(u_{1}\right)-R\left(u_{2}\right), v\right\rangle= & \int_{I}\left[-\frac{1}{2} \phi^{\prime \prime}\left(u_{1 x}(s)\right) u_{1 x x}(s)+\frac{1}{2} \phi^{\prime \prime}\left(u_{2 x}(s)\right) u_{2 x x}(s)\right] v(s) d s \\
= & 2 \int_{I}\left[1-3 u_{1 x}^{2}(s)\right] u_{1 x x}(s) v(s) d s-2 \int_{I}\left[1-3 u_{2 x}^{2}(s)\right] u_{2 x x}(s) v(s) d s \\
= & 2 \int_{I}\left(u_{x x}(s)\right) v(s) d s+6 \int_{I} u_{1 x}^{2}(s) u_{x x}(s) v(s) d s \\
& +6 \int_{I} u_{x}(s) u_{2 x x}(s) v(s)\left[u_{1 x}(s)+u_{2 x}(s)\right] d s \\
\leq & 2\left\|u_{x x}\right\|_{L^{2}(I)}\|v\|_{L^{2}(I)}+6\left\|u_{x x}\right\|_{L^{2}(I)}\|v\|_{L^{2}(I)}\left\|u_{1 x}\right\|_{L^{\infty}(I)}^{2} \\
& \quad+6\left\|u_{2 x x}\right\|_{L^{2}(I)}\left(\int_{I} u_{x}^{2} v^{2}\left(u_{1 x}+u_{2 x}\right)^{2} d s\right)^{1 / 2} \\
\leq & {\left[2+6\left(r_{1} r_{2}\right)^{1 / 2}\right]\|u\|_{H^{2}(I)}\|v\|_{L^{2}(I)}+12 r_{2}\left(r_{1} r_{2}\right)^{1 / 2}\left\|u_{x}\right\|_{L^{\infty}(I)}\|v\|_{L^{2}(I)} } \\
\leq & {\left[2+\left(6+12 r_{2}\right)\left(r_{1} r_{2}\right)^{1 / 2}\right]\|u\|_{H^{2}(I)}\|v\|_{L^{2}(I)}, }
\end{aligned}
$$

where the last inequality follows from (see [19])

$$
\left\|u_{x}\right\|_{L^{\infty}(I)} \leq\left\|u_{x}\right\|_{H^{1}(I)} \leq C\|u\|_{H^{2}(I)} .
$$

Thus, if we take

$$
C_{L}:=2+\left(6+12 r_{2}\right)\left(r_{1} r_{2}\right)^{1 / 2}
$$

then we have that

$$
\left\langle R\left(u_{1}\right)-R\left(u_{2}\right), v\right\rangle \leq C_{L}\left\|u_{1}-u_{2}\right\|_{H^{2}(I)}\|v\|_{H^{2}(I)},
$$

and it follows that the nonlinear functional $R$ is Lipschitz continuous. From the expression of the radii $r_{1}, r_{2}$, it results that the Lipschitz constant is of order $O\left(\varepsilon^{-18}\right)$.

\subsection{Spectral Gap Condition}

Theorem 3.4. There exists a natural number $N$ sufficiently large so that

$$
\tilde{\lambda}_{N+1}-\tilde{\lambda}_{N}>2 C_{L}\left(\tilde{\lambda}_{N+1}^{1 / 2}+\tilde{\lambda}_{N}^{1 / 2}\right)
$$


Proof. The eigenvalues of the operator $A$ are given by

$$
\lambda_{1}=\frac{(2 \pi)^{4}}{|I|^{4}}, \ldots, \lambda_{N}=\lambda_{1} N^{4}
$$

while the eigenvalues of $\widetilde{A}=\varepsilon^{2} A$ are $\tilde{\lambda}_{i}=\varepsilon^{2} \lambda_{i}$. Then the spectral gap condition is

$$
\varepsilon^{2}\left(\lambda_{N+1}-\lambda_{N}\right)>2 C_{L} \varepsilon\left(\lambda_{N+1}^{1 / 2}+\lambda_{N}^{1 / 2}\right) .
$$

In details we have

$$
\varepsilon \mathcal{\lambda}_{1}\left[(N+1)^{4}-N^{4}\right]>2 C_{L} \lambda_{1}^{1 / 2}\left[(N+1)^{2}+N^{2}\right]
$$

that is,

$$
2 N+1>\frac{2 C_{L}}{\varepsilon \lambda_{1}^{1 / 2}}=\frac{C_{L}|I|^{2}}{2 \varepsilon \pi^{2}} .
$$

Then if $N$ satisfies the previous inequality, we have that

$$
\tilde{\lambda}_{N+1}-\tilde{\lambda}_{N}>2 C_{L}\left(\tilde{\lambda}_{N+1}^{1 / 2}+\tilde{\lambda}_{N}^{1 / 2}\right)
$$

Thus, the spectral gap condition is satisfied and the existence of the inertial manifold is assured. This proves Theorem 3.1.

We remark that the dimension of $\mathcal{M}_{\varepsilon}$ is

$$
N \sim C_{L}|I|^{2} \varepsilon^{-1}=O\left(\varepsilon^{-19}\right)
$$

Then comparing the estimates obtained, we have that we get $O\left(\varepsilon^{-1}\right)$ by the method of $n$ dimensional volume evolution and, respectively, $O\left(\varepsilon^{-10}\right)$ and $O\left(\varepsilon^{-19}\right)$ by the existence of the inertial set $\supset_{\varepsilon}$ and the inertial manifold $\mathcal{M}_{\varepsilon}$. It is not a surprise that the dimension of the inertial manifold is much bigger than that of the inertial set and global attractor since we are requiring the existence of a smooth structure.

\section{Regularity of Attractor and Dimension}

In this section we show a result that gives further regularity of the attractor. By a classic theorem (see $[17,20])$, we have the following theorem.

Theorem 4.1. If I is a bounded $C^{\infty}$ domain and if $u_{0}$ and $\Phi$ are $C^{\infty}$ functions, then the global attractor $\mathcal{A}_{\varepsilon}$ is a bounded subset of $H^{k}(I)$ for every $k \geq 0$. In particular, if $u \in \mathcal{A}_{\varepsilon}$, then $u \in C^{\infty}(I)$. 
This regularity result let us conclude by theorem 15.1 in [17] that for $k \geq 16 d_{f}\left(\mathcal{A}_{\varepsilon}\right)+1$, for almost every set $x=\left(x_{1}, \ldots, x_{k}\right)$ of $k$ points in $I$, the mapping

$$
u \rightarrow\left(u\left(x_{1}\right), \ldots, u\left(x_{k}\right)\right)
$$

is an embedding of $\mathcal{A}_{\varepsilon}$ into $\mathbb{R}^{k}$. This gives a parametrization of the attractor $\mathcal{A}_{\varepsilon}$ and gives an estimate of the degree of freedom of the system: $k=O\left(\varepsilon^{-1}\right)$. Consequently if we want to parameterize the attractor by $k$ equally spaced points in $I$, it results that each subinterval of $I$ is of order $|I| / k=O(\varepsilon)$ (see [17] for a more detailed discussion). This is in full accordance with the numerical experiments proposed in [14] in which it was pointed out that the wave length of microstructure is of order $O(\varepsilon)$.

This result has a connection with the definition of determining nodes (see [21]): a set of points $\left\{x_{1}, \ldots, x_{k}\right\}$ in $I$ is said to be asymptotically determining if for two solutions $u_{1}(x, t)$ and $u_{2}(x, t)$

$$
\max _{i=1, \ldots, k}\left|u_{1}\left(x_{i}, t\right)-u_{2}\left(x_{i}, t\right)\right| \longrightarrow 0 \quad \text { as } t \longrightarrow \infty
$$

implies that

$$
\sup _{x \in I}\left|u_{1}(x, t)-u_{2}(x, t)\right| \longrightarrow 0 \quad \text { as } t \longrightarrow \infty
$$

From a theorem in [17], if $A_{\varepsilon}$ attracts all the solutions in the norm of $L^{\infty}(I)$ then almost every set of $k$ nodes $\left\{x_{1}, \ldots, x_{k}\right\}$ in $I$ is asymptotically determining. In the last section we will show that this is true for (1.1); that is, there exists an absorbing set in $L^{\infty}(I)$.

We conclude this section by proving and giving explicit bounds for $A_{\varepsilon}$ in $H^{4}(I)$. In order to do that, we follow a strategy suggested by Robinson in [2].

Theorem 4.2. The global attractor $\mathcal{A}_{\varepsilon}$ of $(1.1)$ is bounded in $H^{4}(I)$.

Proof. Multiply the equation by $u_{t}$ and integrate over $I$ :

$$
\left\|u_{t}\right\|^{2}+\frac{\varepsilon^{2}}{2} \frac{d}{d t}\left\|u_{x x}\right\|^{2}+\frac{1}{2} \int_{I} \Phi^{\prime}\left(u_{x}\right) u_{t x} d x=0
$$

By integrating the previous equation, with respect to time, over $(0, t)$ we get

$$
\int_{0}^{t}\left\|u_{t}\right\|^{2} d s+\frac{\varepsilon^{2}}{2}\left\|u_{x x}(t)\right\|^{2}-\frac{\varepsilon^{2}}{2}\left\|u_{x x}(0)\right\|^{2}+\frac{1}{2} \int_{0}^{t}\left(\int_{I} \Phi^{\prime}\left(u_{x}\right) u_{t x} d x\right) d s=0 .
$$

The integrand of the last term of the previous equality can be written as $(d / d t) \Phi\left(u_{x}\right)$, and this yields to

$$
\int_{0}^{t}\left\|u_{t}\right\|^{2} d s=\frac{\varepsilon^{2}}{2}\left\|u_{x x}(0)\right\|^{2}-\frac{\varepsilon^{2}}{2}\left\|u_{x x}(t)\right\|^{2}+\frac{1}{2} \int_{I}\left[\Phi\left(u_{x}(0)\right)-\Phi\left(u_{x}(t)\right)\right] d x .
$$


Since the attractor is bounded in $L^{\infty}$ and $H^{2}(I)$, we have

$$
\begin{aligned}
\int_{0}^{t}\left\|u_{t}\right\|^{2} d s & \leq \varepsilon^{2} r_{2}^{2}+2|I|\left\|u_{x}\right\|_{L^{\infty}(I)}^{2}\left(\left\|u_{x}\right\|_{L^{\infty}(I)}^{2}+1\right) \\
& \leq \varepsilon^{2} r_{2}^{2}+2|I| r_{1} r_{2}\left(r_{1} r_{2}+1\right):=R .
\end{aligned}
$$

We will prove that $u_{t}$ is uniformly bounded in $L^{2}(I)$. We derive (1.1) with respect to time, multiply it by $t^{2} u_{t}$, and integrate over $I$ :

$$
\begin{aligned}
\frac{1}{2} \frac{d}{d t}\left\|t u_{t}\right\|^{2}-t\left\|u_{t}\right\|^{2}+t^{2} \varepsilon^{2}\left\|u_{x x t}\right\|^{2} & =\frac{t^{2}}{2} \int_{I}\left[\Phi^{\prime}\left(u_{x}\right)\right]_{x t} u_{t} d x \\
& =-\frac{t^{2}}{2} \int_{I} \Phi^{\prime \prime}\left(u_{x}\right) u_{t x}^{2} d x=-6 t^{2} \int_{I} u_{x}^{2} u_{t x}^{2} d x+2 t^{2} \int_{I} u_{t x}^{2} d x
\end{aligned}
$$

Then, using interpolation we get

$$
\frac{1}{2} \frac{d}{d t}\left\|t u_{t}\right\|^{2}-t\left\|u_{t}\right\|^{2}+t^{2} \varepsilon^{2}\left\|u_{x x t}\right\|^{2} \leq 2 t^{2}\left\|u_{t x}\right\|^{2} \leq t^{2} \varepsilon^{2}\left\|u_{x x t}\right\|^{2}+\frac{t^{2}}{\varepsilon^{2}}\left\|u_{t}\right\|^{2},
$$

from which

$$
\frac{1}{2} \frac{d}{d t}\left\|t u_{t}\right\|^{2} \leq \frac{t^{2}}{\varepsilon^{2}}\left\|u_{t}\right\|^{2}+t\left\|u_{t}\right\|^{2}
$$

Integrating the previous inequality, with respect to time, in $(0, t)$, we have

$$
\left\|t u_{t}\right\|^{2} \leq 2 \int_{0}^{t}\left(s+\frac{s^{2}}{\varepsilon^{2}}\right)\left\|u_{t}\right\|^{2} d s
$$

Since the term $\left(s+s^{2} / \varepsilon^{2}\right)$ is bounded in $[0,1]$, we obtain the following inequality by setting $t=1$ :

$$
\left\|u_{t}(1)\right\|^{2} \leq 2\left(1+\frac{1}{\varepsilon^{2}}\right) \int_{0}^{t}\left\|u_{t}\right\|^{2} d s \leq 2 R\left(1+\frac{1}{\varepsilon^{2}}\right) .
$$

This gives an uniform $L^{2}$ bound for $u_{t}(1)$, and since $u \in \mathcal{A}_{\varepsilon}$, it is sufficient to conclude that $u_{t} \in L^{\infty}\left(\mathbb{R}, L^{2}(I)\right)$. In particular, let $u \in \mathcal{A}_{\varepsilon}$ and $s \geq 0$, we set $\widehat{u}(t, x):=u(s+t-1, x)$ for any $t \geq 1$. Then $\widehat{u}_{t}(t, x)=u_{t}(s+t-1, x)$ and so $\widehat{u}_{t}(1, x)=u_{t}(s, x)$, from which we have that

$$
\left\|u_{t}(s)\right\|^{2} \leq 2 R\left(1+\frac{1}{\varepsilon^{2}}\right), \quad \forall s>0 .
$$


Now we consider the $L^{2}$ norm of the nonlinear term $(1 / 2) \Phi^{\prime \prime}\left(u_{x}\right) u_{x x}$ :

$$
\begin{aligned}
\frac{1}{4} \int_{I}\left(\Phi^{\prime \prime}\left(u_{x}\right)\right)^{2} u_{x x}^{2} d x & \leq 4 \int_{I}\left(\sqrt{3}\left\|u_{x}\right\|_{L^{\infty}(I)}+1\right)^{2} u_{x x}^{2} d x \\
& \leq 4\left(6\left\|u_{x}\right\|_{L^{\infty}(I)}^{2}+1\right)\left\|u_{x x}\right\|^{2} \leq 4\left(6 r_{1} r_{2}+1\right) r_{2}^{2},
\end{aligned}
$$

where we have used (1.8), (1.10), and [15]. Then, from the previous inequality, we get that $1 / 2 \Phi^{\prime \prime}\left(u_{x}\right) u_{x} x$ is uniformly bounded in $L^{2}(I)$. Then since

$$
\varepsilon^{2} u_{x x x x}=-u_{t}+\frac{1}{2} \Phi^{\prime \prime}\left(u_{x}\right) u_{x x}
$$

we have that $u_{x x x x}$ is uniformly bounded in $L^{2}(I)$. Then from the following interpolating inequality

$$
\left\|u_{x x x}\right\| \leq\left\|u_{x x}\right\|^{1 / 2}\left\|u_{x x x x}\right\|^{1 / 2}
$$

we get that $\mathscr{A}_{\varepsilon}$ is bounded in $H^{4}(I)$.

\section{Absorbing Set in $L^{\infty}(I)$}

In this last section we show the existence of an absorbing set in $L^{\infty}(I)$.

Theorem 5.1. The semigroup $S(t)$ defined in (1.4) posses an absorbing set in $L^{\infty}(I)$ :

$$
\left\|S(t) u_{0}\right\|_{L^{\infty}(I)} \leq r_{\infty} \text {, if } t>\tau\left(R_{\infty}\right)+\varepsilon^{2},
$$

where

$$
r_{\infty}=\left(1+\frac{1}{\varepsilon^{2}}\right)^{1 / 2} \frac{|I|^{1 / 2}}{\varepsilon^{1 / 2}}\left\{\frac{\varepsilon^{2}}{2}\left(1+\frac{4}{\varepsilon^{4}}\right)+\frac{1}{\varepsilon^{2}}\right\}^{1 / 4}, \quad \tau\left(R_{\infty}\right)=\frac{1}{\varepsilon^{2}} \log R_{\infty}
$$

and $R_{\infty}$ is a positive constant such that

$$
\left\|u_{0}\right\|_{L^{\infty}(I)} \leq R_{\infty} .
$$

Proof. Let $u$ be a solution of (1.1) such that $u(0)=u_{0} \in L^{\infty}(I)$ and $\left\|u_{0}\right\|_{L^{\infty}(I)} \leq R_{\infty}$. Multiply (1.1) by $u$ and integrate on $I$, we obtain

$$
\frac{1}{2} \frac{d}{d t}\|u\|^{2}+\varepsilon^{2}\left\|u_{x x}\right\|^{2}+\frac{1}{2} \int_{I} \Phi^{\prime}\left(u_{x}\right) u_{x} d x=0
$$


from which

$$
\frac{1}{2} \frac{d}{d t}\|u\|^{2}+\varepsilon^{2}\left\|u_{x x}\right\|^{2}+2\left\|u_{x}\right\|_{L^{4}(I)}^{4}=2\left\|u_{x}\right\|^{2} \leq 2\left\|u_{x}\right\|_{L^{4}(I)}^{4}|I|^{1 / 2} \leq\left\|u_{x}\right\|_{L^{4}(I)}^{4}+|I|,
$$

and then

$$
\frac{1}{2} \frac{d}{d t}\|u\|^{2}+\varepsilon^{2}\left\|u_{x x}\right\|^{2} \leq|I|
$$

By the boundary conditions, from the hypothesis that $|I| \leq 1$ and from the interpolating inequality $\left\|u_{x}\right\|^{2} \leq(1 / 2)\left\{\|u\|^{2}+\left\|u_{x x}\right\|^{2}\right\}$, we have that

$$
\|u\| \leq\left\|u_{x x}\right\| .
$$

Then we can rewrite (5.6) only in terms of the $L^{2}$-norm of $u$ :

$$
\frac{1}{2} \frac{d}{d t}\|u\|^{2}+\varepsilon^{2}\|u\|^{2} \leq|I|
$$

Integrating the previous inequality over $(0, t)$, we get

$$
\|u(t)\|^{2} \leq\left\|u_{0}\right\|^{2} e^{-2 \varepsilon^{2} t}+\frac{|I|}{\varepsilon^{2}}\left(1-e^{-2 \varepsilon^{2} t}\right) \leq\left(R_{\infty}^{2} e^{-2 \varepsilon^{2} t}+\frac{1}{\varepsilon^{2}}\right)|I|,
$$

where the last inequality follows from $\|u\| \leq|I|^{1 / 2}\|u\|_{L^{\infty}(I)}$. Then if we set

$$
\tau\left(R_{\infty}\right)=\frac{1}{\varepsilon^{2}} \log R_{\infty}
$$

we have that

$$
\|u(t)\|^{2} \leq\left(1+\frac{1}{\varepsilon^{2}}\right)|I|, \quad \text { if } t \geq \tau\left(R_{\infty}\right) .
$$

Now coming back to (5.5), we use a different estimate method

$$
\frac{1}{2} \frac{d}{d t}\|u\|^{2}+\varepsilon^{2}\left\|u_{x x}\right\|^{2}+2\left\|u_{x}\right\|_{L^{4}(I)}^{4}=2\left\|u_{x}\right\|^{2} \leq 2\|u\|\left\|u_{x x}\right\| \leq \frac{\varepsilon^{2}}{2}\left\|u_{x x}\right\|^{2}+\frac{2}{\varepsilon^{2}}\|u\|^{2}
$$

from which

$$
\frac{d}{d t}\|u\|^{2}+\varepsilon^{2}\left\|u_{x x}\right\|^{2} \leq \frac{4}{\varepsilon^{2}}\|u\|^{2}
$$


Integrating the previous inequality with respect to time in $(t, t+\rho)$, with $\rho \geq \tau\left(R_{\infty}\right)$, we get

$$
\varepsilon^{2} \int_{t}^{t+\rho}\left\|u_{x x}\right\|^{2} d t \leq\left(4 / \varepsilon^{2}+2\right)\|u\|^{2} \leq\left(4 / \varepsilon^{2} \rho+2\right)\left(1+1 / \varepsilon^{2}\right)|I| .
$$

Moreover by an interpolating inequality, we have that

$$
\begin{aligned}
\int_{t}^{t+\rho}\|u\|^{2} d t & \leq \frac{1}{2}\left\{\int_{t}^{t+\rho}\|u\|^{2}+\int_{t}^{t+\rho}\left\|u_{x x}\right\|^{2}\right\} \\
& \leq\left(1+\frac{1}{\varepsilon^{2}}\right)|I|\left\{\frac{\rho}{2}\left(1+\frac{4}{\varepsilon^{4}}\right)+\frac{1}{\varepsilon^{2}}\right\}:=a_{3} .
\end{aligned}
$$

We multiply (1.1) by $\boldsymbol{u}_{x x}$ and integrate over $I$ :

$$
\frac{1}{2} \frac{d}{d t}\left\|u_{x}\right\|^{2}+\varepsilon^{2}\left\|u_{x x x}\right\|^{2}+6\left\|u_{x}^{2} u_{x x}\right\|^{2}=2\left\|u_{x x}\right\|^{2}
$$

from which by using an interpolating inequality and neglecting the positive term $6\left\|u_{x}^{2} u_{x x}\right\|^{2}$ we get

$$
\frac{1}{2} \frac{d}{d t}\left\|u_{x}\right\|^{2}+\varepsilon^{2}\left\|u_{x x x}\right\|^{2} \leq \frac{1}{\varepsilon^{2}}\left\|u_{x}\right\|^{2}+\varepsilon^{2}\left\|u_{x x x}\right\|^{2}
$$

from which we conclude

$$
\frac{d}{d t}\left\|u_{x}\right\|^{2} \leq \frac{2}{\varepsilon^{2}}\left\|u_{x}\right\|^{2} .
$$

We apply the uniform Gronwall lemma (see [1]) with

$$
a_{1}=\int_{t}^{t+\rho} \frac{2}{\varepsilon^{2}} d s=\frac{2 \rho}{\varepsilon^{2}}, \quad a_{2}=0,
$$

from which we obtain

$$
\left\|u_{x}(t+\rho)\right\|^{2} \leq \frac{a_{3}}{\rho} e^{a_{1}}, \quad \text { for } t \geq \tau\left(R_{\infty}\right) .
$$

We fix $\rho:=\varepsilon^{2}$, and then

$$
\left\|u_{x}\right\| \leq\left(1+\frac{1}{\varepsilon^{2}}\right)^{1 / 2} \frac{|I|^{1 / 2}}{\varepsilon}\left\{\frac{\varepsilon^{2}}{2}\left(1+\frac{4}{\varepsilon^{4}}\right)+\frac{1}{\varepsilon^{2}}\right\}^{1 / 2}, \quad \text { for } t \geq \tau\left(R_{\infty}\right)+\varepsilon^{2} .
$$


Then, using an interpolating inequality, we have that

$$
\|u\|_{L^{\infty}(I)} \leq\|u\|^{1 / 2}\left\|u_{x}\right\|^{1 / 2} \leq r_{\infty}, \quad \text { for } t \geq \tau\left(R_{\infty}\right)+\varepsilon^{2},
$$

where

$$
r_{\infty}=\left(1+\frac{1}{\varepsilon^{2}}\right)^{1 / 2} \frac{|I|^{1 / 2}}{\varepsilon^{1 / 2}}\left\{\frac{\varepsilon^{2}}{2}\left(1+\frac{4}{\varepsilon^{4}}\right)+\frac{1}{\varepsilon^{2}}\right\}^{1 / 4} .
$$

This completes the proof, the set

$$
B_{\infty}=\left\{u \in L^{\infty}(I):\|u\|_{L^{\infty}(I)} \leq r_{\infty}\right\}
$$

is absorbing for all the bounded sets of $L^{\infty}(I)$.

\section{Acknowledgment}

The first author would like to thank Professor Giorgio Fusco for suggesting the subject of research during his Ph.D. studies and for helpful hints and remarks.

\section{References}

[1] R. Temam, Infinite-Dimensional Dynamical Systems in Mechanics and Physics, vol. 68, Springer, New York, NY, USA, 2nd edition, 1997.

[2] J. C. Robinson, Infinite-Dimensional Dynamical Systems: An Introduction to Dissipative Parabolic PDEs and the Theory of Global Attractors, Cambridge Texts in Applied Mathematics, Cambridge University Press, Cambridge, UK, 2001.

[3] L. Yang and M.-H. Yang, "Attractors of the non-autonomous reaction-diffusion equation with nonlinear boundary condition," Nonlinear Analysis, vol. 11, no. 5, pp. 3946-3954, 2010.

[4] T. Caraballo, G. Łukaszewicz, and J. Real, "Pullback attractors for asymptotically compact nonautonomous dynamical systems," Nonlinear Analysis, vol. 64, no. 3, pp. 484-498, 2006.

[5] A. Tarasińska, "Pullback attractor for heat convection problem in a micropolar fluid," Nonlinear Analysis, vol. 11, no. 3, pp. 1458-1471, 2010.

[6] M. Yang and P. E. Kloeden, "Random attractors for stochastic semi-linear degenerate parabolic equations," Nonlinear Analysis, vol. 12, no. 5, pp. 2811-2821, 2011.

[7] Z. Wang, S. Zhou, and A. Gu, "Random attractor for a stochastic damped wave equation with multiplicative noise on unbounded domains," Nonlinear Analysis, vol. 12, no. 6, pp. 3468-3482, 2011.

[8] T. Caraballo, J. A. Langa, V. S. Melnik, and J. Valero, "Pullback attractors of nonautonomous and stochastic multivalued dynamical systems," Set-Valued Analysis, vol. 11, no. 2, pp. 153-201, 2003.

[9] O. V. Kapustyan, P. O. Kasyanov, and J. Valero, "Pullback attractors for a class of extremal solutions of the 3D Navier-Stokes system," Journal of Mathematical Analysis and Applications, vol. 373, no. 2, pp. 535-547, 2011.

[10] N. Saito, "Conservative upwind finite-element method for a simplified Keller-Segel system modelling chemotaxis," IMA Journal of Numerical Analysis, vol. 27, no. 2, pp. 332-365, 2007.

[11] M. Efendiev, E. Nakaguchi, and W. L. Wendland, "Dimension estimate of the global attractor for a semi-discretized chemotaxis-growth system by conservative upwind finite-element scheme," Journal of Mathematical Analysis and Applications, vol. 358, no. 1, pp. 136-147, 2009.

[12] A. Eden, C. Foias, B. Nicolaenko, and R. Temam, Exponential Attractors for Dissipative Evolution Equations, vol. 37, John Wiley \& Sons, 1995. 
[13] M. Bulíček and D. Pražák, "A note on the dimension of the global attractor for an abstract semilinear hyperbolic problem," Applied Mathematics Letters, vol. 22, no. 7, pp. 1025-1028, 2009.

[14] G. Bellettini, G. Fusco, and N. Guglielmi, "A concept of solution and numerical experiments for forward-backward diffusion equations," Discrete and Continuous Dynamical Systems A, vol. 16, no. 4, pp. 783-842, 2006.

[15] G. R. Chacón and R. Colucci, "Asymptotic behavior of a fourth order evolution equation," submitted paper.

[16] P. Constantin, C. Foias, B. Nicolaenko, and R. Temam, Integral Manifolds and Inertial Manifolds for Dissipative Partial Differential Equations, vol. 70 of Applied Mathematical Sciences, Springer, New York, NY, USA, 1st edition, 1988.

[17] J. C. Robinson, Dimensions, Embeddings, and Attractors, vol. 186 of Cambridge Texts in Applied Mathematics, Cambridge University Press, Cambridge, UK, 2011.

[18] M. Slemrod, "Dynamics of measure valued solutions to a backward-forward heat equation," Journal of Dynamics and Differential Equations, vol. 3, no. 1, pp. 1-28, 1991.

[19] H. Brezis, Functional Analysis, Sobolev Spaces and Partial Differential Equations, Springer, New York, NY, USA, 2010.

[20] R. Mañé, "On the dimension of the compact invariant sets of certain nonlinear maps," in Dynamical Systems and Turbulence, vol. 898 of Lecture Notes in Mathematics, pp. 230-242, Springer, Berlin, Germany, 1981.

[21] C. Foias and R. Temam, "Determination of the solutions of the Navier-Stokes equations by a set of nodal values," Mathematics of Computation, vol. 43, no. 167, pp. 117-133, 1984. 


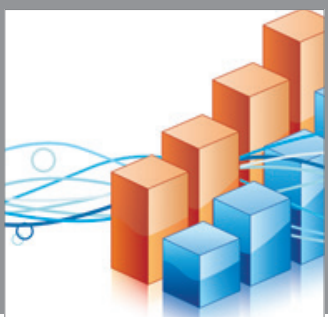

Advances in

Operations Research

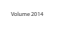

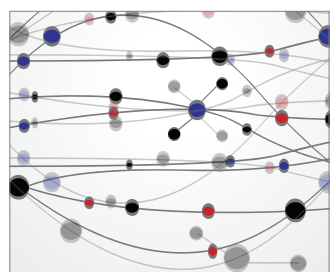

\section{The Scientific} World Journal
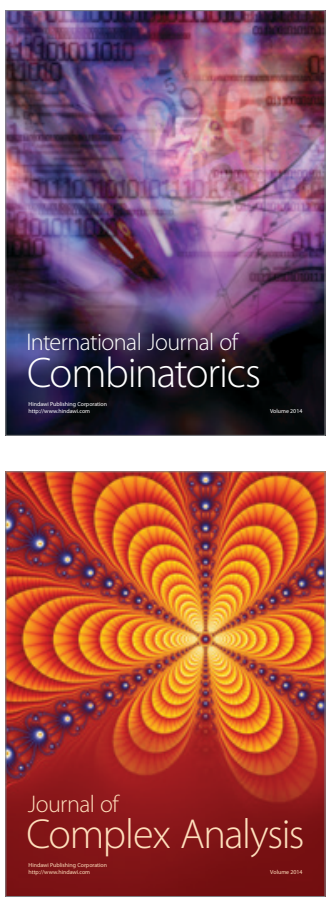

International Journal of

Mathematics and

Mathematical

Sciences
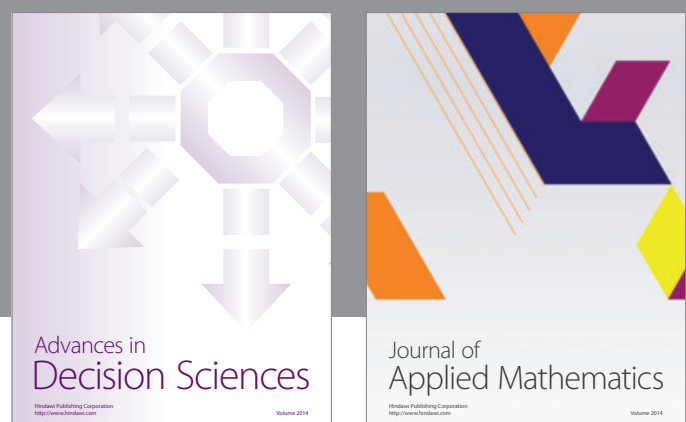

Journal of

Applied Mathematics
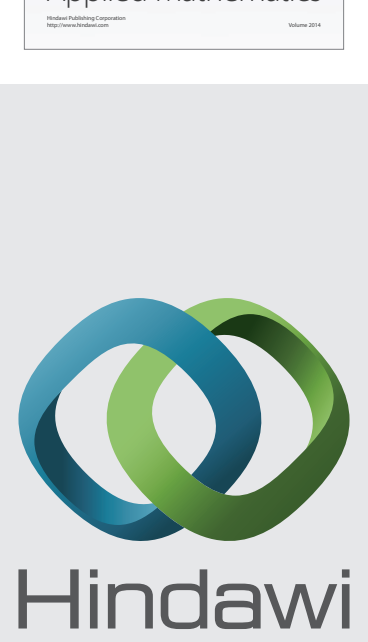

Submit your manuscripts at http://www.hindawi.com
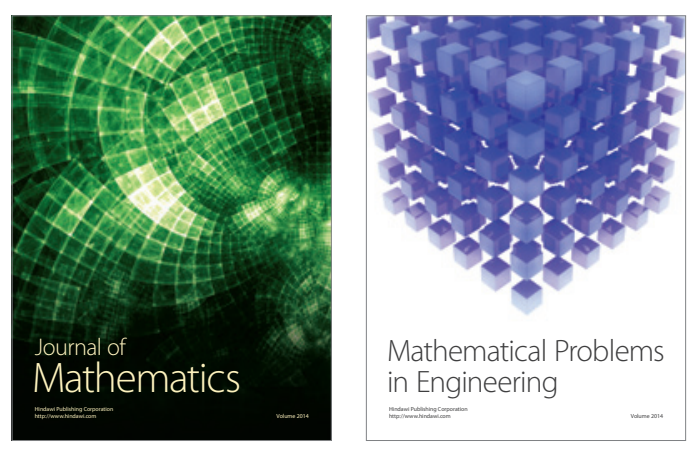

Mathematical Problems in Engineering
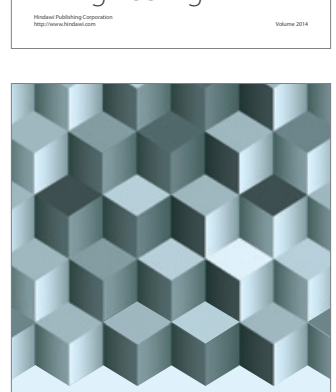

Journal of

Function Spaces
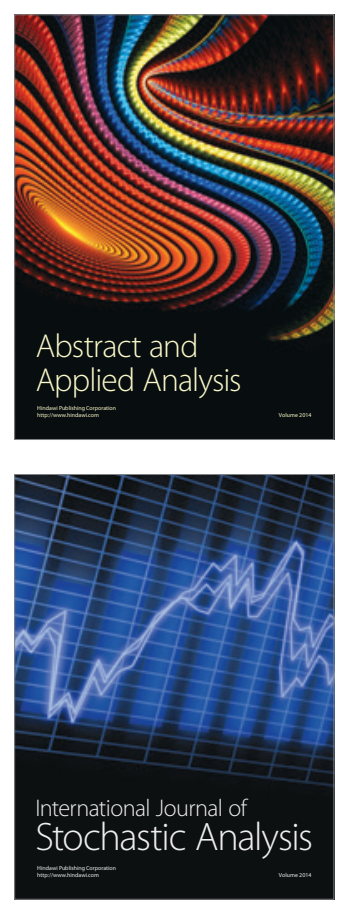

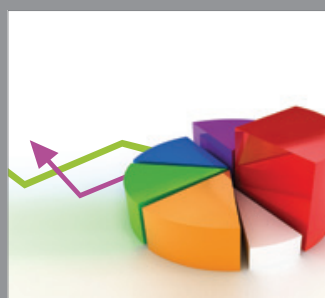

ournal of

Probability and Statistics

Promensencen
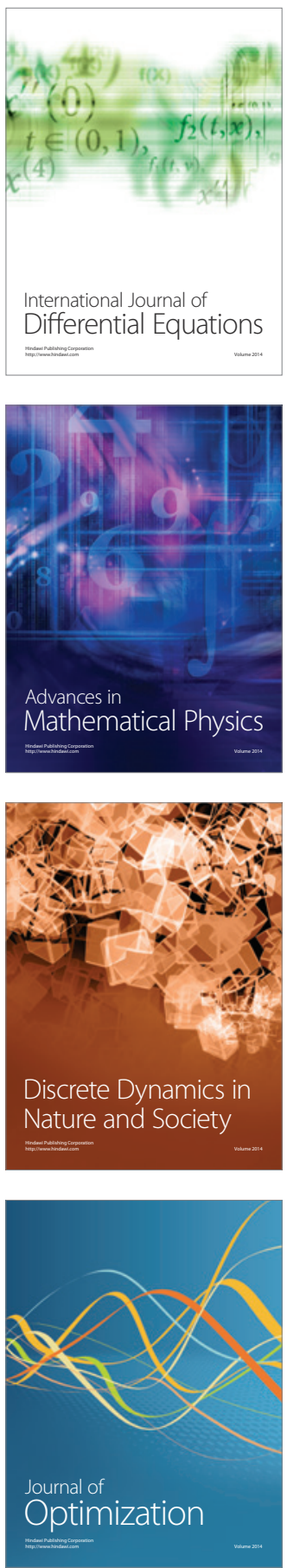